\title{
RELATING TO THE PROOF OF AN EXISTENCE THEOREM FOR A CERTAIN TYPE OF BOUNDARY VALUE PROBLEM*
}

BY H. T. DAVIS

1. Introduction. The problem of proving the existence of a solution of an ordinary, linear, homogeneous differential equation of $n$th order which satisfies $n$ linear, homogeneous boundary conditions of $(n-1)$ st order at $p$ points has been attacked in various ways since it was first formulated by Sturm in 1836. $\dagger$ One of the most fruitful of these methods was that first employed by Bôcher, $\ddagger$ and later applied by Birkhoff § and Ettlinger $\uparrow$ to general self-adjoint systems of second order. This method is essentially the study of the zeros of a certain determinant of $n$th order by means of the properties of a system for which the existence of solutions has already been established.

The object of the present paper is to consider the problem by means of integral equations, from which is obtained a criterion of some elegance, although the generality of this method is somewhat limited by the way in which the parameter enters the integral equation.

2. Theorems to be used. Suppose $L(u)$ is any linear, homogeneous, ordinary differential expression of $n$th order and suppose the coefficient of $u^{(n)}\left(p_{0}\right)$ is different from zero in the interval $a \leqq x \leqq b$. Consider, then, the two systems:

(I) $\quad\left\{\begin{aligned} L(u)+\lambda u & =0 \\ U_{i}(u) & =0\end{aligned}\right.$

$$
\left\{\begin{aligned}
L(u)+\lambda u & =0 \\
V_{i}(u) & =0,
\end{aligned}\right.
$$

$(i=1,2,3, \cdots, n)$, where $U_{i}$ and $V_{i}$ are linear combinations of $u$ and its first $(n-1)$ derivatives at the two points $a$ and $b$.

* Presented to the Society, April 15, 1922.

† Journal de Mathématiques, vol. 1 (1836), pp. 106-186.

† Comptes Rendus, vol. 140 (1905), p. 928.

§ Transactions of this Society, vol. 10 (1909), pp. 259-270.

I Transactions of this Society, vol. 19 (1918), pp. 79-96. 
We shall suppose that the existence of an infinite sequence of $\lambda$ 's $\left(\lambda_{1}, \lambda_{2}, \cdots\right)$ is known for system I. Our object is to show how this fact may be used to find conditions upon the coefficients of system II which will insure the existence of a sequence of $\lambda$ 's $\left(l_{1}, l_{2}, \cdots\right)$ for this system also.

We make use of the following theorem in the theory of integral equations:

If the Green's function of the differential expression $L(u)$ for such a set of boundary conditions as I or II is taken as the kernel of an integral equation of the second kind,

$$
f(x)=\phi(x)-\lambda \int_{a}^{b} K(x, t) \phi(t) d t,
$$

the Green's function of the differential expression $L(u)+\lambda u$ which corresponds to the boundary conditions I or II is the resolvant function $K(x, t)$ of this integral equation.*

We recall that if

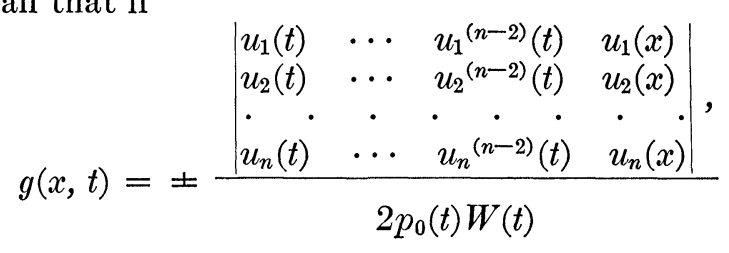

where $W(t)$ is the Wronskian of the fundamental set of solutions, $u_{1}, \cdots, u_{n}$, of the equation $L(u)=0$, and their derivatives to the $(n-1)$ st order, and where the plus sign refers to $x \leqq t$, and the minus sign to $x \geqq t$, then

$$
G(x, t)=g(x, t)+c_{1} u_{1}(x)+\cdots+c_{n} u_{n}(x)
$$

is the Green's function of $L(u)$ provided $c_{1}, \cdots, c_{n}$ are so chosen that $G(x, t)$ will satisfy the boundary conditions $U_{i}(u)=0$.

We notice that $G(x, t)$ exists and is a continuous function

* Hilbert, Grundzüge einer allgemeiner Theorie der linearen Integralgleichungen, Teubner, 1912, p. 49. Here the theorem is proved only for the special self-adjoint case of second order, but a consideration shows that the same proof may be extended to include the case of a system of $n$th order. 
in both arguments except when

$$
F(\lambda)=\left|\begin{array}{ccc}
U_{1}\left(u_{1}\right) & \cdots & U_{1}\left(u_{n}\right) \\
\cdot & \cdot & \cdot \\
U_{n}\left(u_{1}\right) & \cdots & U_{n}\left(u_{n}\right)
\end{array}\right|=0 .
$$

3. Interrelation of Problems I and II. We shall now suppose that $\Gamma_{1}(x, t)$ and $\Gamma_{2}(x, t)$ are the Green's functions of $L(u)+\lambda u$ for boundary conditions I and II, and that $K_{1}(x, t)$ and $K_{2}(x, t)$ are the Green's functions of $L(u)$ for the same boundary conditions. Consequently $\Gamma_{1}$ and $\Gamma_{2}$ are the resolvant functions for the integral equations

$$
u(x)=\lambda \int_{a}^{b} K_{1}(x, t) u(t) d t, \quad u(x)=\lambda \int_{a}^{b} K_{2}(x, t) u(t) d t,
$$

and we are led to consider the two Fredholm determinants $D_{1}$ and $D_{2}$ which belong to problems I and II.

By the well known property of these determinants, ${ }^{*}$ we have:

$$
\frac{d D_{1}}{d \lambda}=-D_{1} \int_{a}^{b} \Gamma_{1}(x, x) d x, \quad \frac{d D_{2}}{d \lambda}=-D_{2} \int_{a}^{b} \Gamma_{2}(x, x) d x .
$$

Since the zeros of $D_{1}(\lambda)$ and $D_{2}(\lambda)$ are the characteristic values $\left(\lambda_{1}, \lambda_{2}, \cdots\right)$ and $\left(l_{1}, l_{2}, \cdots\right)$ for which solutions of I and II exist, we consider the ratio $D_{1} / D_{2}$. Between any two characteristic values $\lambda_{i}$ and $\lambda_{i+1}, D_{1} / D_{2}$ is a continuous function providing $D_{2}$ does not vanish in the interval. Hence its derivative must vanish in the interval:

$$
\begin{aligned}
\frac{d}{d \lambda}\left(\frac{D_{1}}{D_{2}}\right)= & \frac{D_{2} \frac{d D_{1}}{d \lambda}-D_{1} \frac{d D_{2}}{d \lambda}}{D_{2}^{2}} \\
= & \frac{-D_{2} D_{1} \int_{a}^{b} \Gamma_{1} d x+D_{1} D_{2} \int_{a}^{b} \Gamma_{2} d x}{D_{2}^{2}} \\
= & -\frac{D_{1}}{D_{2}} \int_{a}^{b}\left[\Gamma_{1}(x, x)-\Gamma_{2}(x, x)\right] d x .
\end{aligned}
$$

* Hilbert, loc. cit., pp. 12-13. 
But suppose that it is shown that $\boldsymbol{S}_{a}^{b}\left(\Gamma_{1}-\Gamma_{2}\right) d x \neq 0$ for $\lambda_{i} \leqq \lambda \leqq \lambda_{i+1}$. Our hypothesis that $D_{1} / D_{2}$ is a continuous function is contradicted. Consequently $D_{2}$ must vanish in the interval. This result may be stated in the following theorem.

Theorem. If the existence of a sequence of $\lambda$ 's for which solutions of problem I exist has been established, then a sequence of l's for problem II alternating or coincident with the $\lambda$ 's of problem I exist provided

$$
\int_{a}^{b}\left[\Gamma_{1}(x, x)-\Gamma_{2}(x, x)\right] d x \neq 0, \quad\left(\lambda_{0} \leqq \lambda \leqq \lambda_{m}\right),
$$

where $\Gamma_{1}$ and $\Gamma_{2}$ are the Green's functions belonging to problems I and II.

4. Examples. Two examples serve to illustrate the theorem:

$$
\begin{gathered}
u^{\prime \prime}+\lambda u=0, \\
\text { I. }\left\{\begin{array} { l } 
{ u ( 0 ) = 0 , } \\
{ u ( 1 ) = 0 , }
\end{array} \quad \text { II. } \left\{\begin{array}{c}
u(0)=0, \\
u^{\prime}(1)=0 .
\end{array}\right.\right.
\end{gathered}
$$

Specialize $u_{1}$ and $u_{2}$ by assuming $u_{1}(0)=0, u_{1}^{\prime}(0)=1$; $u_{2}(0)=1, u_{2}^{\prime}(0)=0$. Then

$$
\int_{0}^{1}\left(\Gamma_{1}-\Gamma_{2}\right) d x=-\int_{0}^{1} \frac{u_{1}{ }^{2}(x)}{u_{1}(1) u_{1}{ }^{\prime}(1)} d x
$$

which is never zero. Consequently the characteristic numbers of the two systems must always alternate with one another, a conclusion easily verified by explicitly solving the two systems.

$$
\begin{gathered}
\left(p u^{\prime}\right)^{\prime}+q u+\lambda u=0, \\
\text { I. }\left\{\begin{array} { l } 
{ u ( a ) = 0 , } \\
{ u ( b ) = 0 , }
\end{array} \text { II. } \left\{\begin{array}{r}
u(a)-u(b)=0 . \\
u^{\prime}(a)-u^{\prime}(b)=0 .
\end{array}\right.\right.
\end{gathered}
$$

Again specialize the fundamental system by assuming $u_{1}(a)=0, u_{1}^{\prime}(a)=1, u_{2}(a)=1, u_{2}^{\prime}(a)=0$.

Then

$$
\begin{array}{r}
\int_{a}^{b}\left(\Gamma_{1}-\Gamma_{2}\right) d x=\frac{1}{D_{1} D_{2}} \int_{a}^{b}\left\{\left[u_{2}^{2}(b)-\left(1+p_{1}\right) u_{2}(b)\right.\right. \\
\left.+p_{1}\right] u_{1}^{2}(x)-\left[2 u_{1}(b) u_{2}(b)-\left(p_{1}+1\right) u_{1}(b)\right] u_{1}(x) u_{2}(x) \\
\left.+u_{1}^{2}(b) u_{2}^{2}(x)\right\} d x
\end{array}
$$


where $D_{1}$ and $D_{2}$ are the characteristic functions (2) of the two systems and $p_{1}=p(a) / p(b)$. A sufficient condition, then, that the characteristic numbers of the two systems shall either alternate or coincide, is that the quadratic form in $u_{1}(x), u_{2}(x)$ shall be definite. But the discriminant $\Delta$ of the form is

$$
\Delta=\left(p_{1}-1\right)^{2} u_{1}{ }^{2} .
$$

Consequently the form will be definite if and only if we have $p(a)=p(b)$, which is the well known condition that system II shall be self-adjoint.*

The University of Wisconsin

\section{NOTE CONCERNING THE ROOTS OF AN EQUATION}

BY K. P. WILLIAMS

Professors Carmichael and Mason have published the following theorem. $\dagger$

All roots of the equation

$$
x^{n}+a_{1} x^{n-1}+a_{2} x^{n-2}+\cdots+a_{n}=0
$$

are, in absolute value, less than

$$
\sqrt{1+\left|a_{1}\right|^{2}+\left|a_{2}\right|^{2}+\cdots+\left|a_{n}\right|^{2}} \text {. }
$$

It is apparent that this limit may be greatly in excess of the actual maximum of the absolute values of the roots. An illustration of this fact is furnished by the equation

$$
x^{n}+x^{n-1}+\cdots+x+1=0 .
$$

The theorem asserts that $\sqrt{n+1}$ is greater than the absolute value of any root. If $n$ is large this is rather meager and inexact information, since all roots are in absolute value exactly 1, irrespective of the value of $n$.

* Note on the roots of algebraic equations, this Bulletin, vol. 21 (1914), p. 21.

† This example is treated by Bôcher by different means in his Leçons sur les Methodes de Sturm, 1917, pp. 83-91. 